\title{
Resposta aguda cardio-respiratória a quatro modos de exercício realizado em ergómetros
}

\author{
C.I. Abrantes ${ }^{1}$ \\ J.E. Sampaio ${ }^{1}$ \\ A.M. Reis ${ }^{1}$
}

J.A. Duarte ${ }^{2}$

https://doi.org/10.5628/rpcd.04.01.66

\section{RESUMO}

O objectivo que se colocou para este estudo, surgiu da necessidade de se conhecer se existem diferenças na resposta aguda cardio-respiratória em quatro modos de exercício (tapete, bicicleta, elíptica e remo), para idênticas percentagens de frequência cardíaca. A amostra foi composta por 14 indivíduos do sexo feminino fisicamente activos e saudáveis. Para cada ergómetro, foram realizados três patamares de exercício, cada um com a duração de cinco minutos e com um intervalo de um minuto entre eles, às seguintes intensidades: no primeiro patamar $60 \%$ a $65 \%$ da frequência cardíaca máxima teórica, no segundo patamar $70 \%$ a $75 \%$ e no terceiro patamar de $80 \%$ a $85 \%$. Em cada patamar, foram medidas as seguintes variáveis: consumo de oxigénio relativo, ventilação, quociente respiratório e frequência cardíaca. Nos intervalos entre patamares, foram medidas as tensões arteriais sistólica e diastólica. Os resultados obtidos foram relativizados aos valores de repouso (estabelecido em $100 \%$ ) e foram calculadas equações de regressão para cada variável, em cada um dos ergómetros em estudo. Através de uma ANOVA simples para medidas independentes foram testadas, em cada patamar de exercício, as diferenças nos valores médios de cada variável dependente, em função do modo de exercício. De acordo com os resultados, para a mesma frequência cardíaca, a magnitude das adaptações agudas ao esforço variou em função do modo de exercício. Para cada ergómetro e para cada variável foram encontradas diferenças estatisticamente significativas $(\mathrm{p}<0,05)$ nos patamares de exercício estudados. O perfil de resposta aguda no tapete caracterizou-se pelos maiores valores de consumo de oxigénio relativo e menores valores do quociente respiratório, como consequência provável das diferenças na quantidade de massa muscular envolvida no exercício e do tipo de suporte do peso corporal. Na bicicleta, os valores de tensão arterial sistólica foram os mais elevados e os valores de tensão arterial diastólica foram os mais baixos. $\mathrm{Na}$ elíptica, identificaram-se menores valores de consumo de oxigénio relativo e valores do quociente respiratório mais elevados. No remo, o mesmo esforço apresentou maiores valores da ventilação e a resposta das tensões arteriais (sistólica e diastólica) foi mais ténue. No geral, estas diferenças na resposta aguda parecem depender da quantidade de massa muscular envolvida no exercício, do suporte do peso corporal e do tipo de unidades motoras solicitadas. Apesar dos diversos ergómetros disponíveis permitirem atender ao gosto individual (e promoverem a adesão ao exercício), os cuidados no ajuste da prescrição do exercício são imperativos.

Palavras-chave: resposta aguda cárdio-respiratória, modo de exercício, frequência cardíaca.

\author{
${ }^{1}$ Departamento de Desporto, Universidade de Trás-os- \\ -Montes e Alto Douro, Portugal. \\ ${ }^{2}$ Faculdade de Ciências do Desporto e Educação Física \\ Universidade do Porto, Portugal
}

\begin{abstract}
Acute cardiorespiratory responses to four ergometer exercise modes.
\end{abstract}

The purpose of the present study was to examine the acute cardiorespiratory responses to four exercise modes (treadmill, cycle ergometer, elliptical and rowing ergometer) at identical heart rates. In each ergometer, 14 active healthy females performed three exercise bouts (5 minutes each) with 1 minute rest period between each bout. The first bout was performed at 60-65\% of maximal heart rate, the second at $70-75 \%$ and the third at $80-85 \%$. In each bout, we measured the following variables: relative oxygen uptake, ventilation, respiratory exchange ratio and heart rate. In the rest periods, we measured systolic and diastolic blood pressure. The results were relativised to rest values and regression equations for each variable and ergometer were calculated. One-way ANOVA tested the differences across exercise modes for each exercise bout. According to the results, at the same heart rate, the magnitude of the acute responses changed across all studied exercise modes. In fact, all variables were statistically different $(p<0,05)$ across exercise modes for the three exercise bouts. Treadmill relative oxygen uptake was the highest and respiratory exchange ratio was the lowest, probably due to the differences in active muscle mass and the absence of body mass support. Elliptical relative oxygen uptake values were the lowest and respiratory exchange ratio the highest. Rowing ergometer induced higher ventilation values and lowest systolic and diastolic blood pressure. Globally, these differences seem to be associated to active muscle mass, body mass support and motor unit recruitment. Available ergometers allow attending to individual preferences (and promoting exercise adherence), however regulating exercise prescription according to population characteristics and their specific goals is an imperative need.

KeyWords: exercise mode, heart rate, acute cardiorespiratory response. 


\section{INTRODUÇÃO}

Em função do modo de exercício, existem diferentes perfis de resposta fisiológica ao exercício realizado em ergómetros, fundamentalmente determinados pela utilização de membros inferiores (MI), membros superiores (MS) ou utilização simultânea de MI e MS, pelo suporte do peso corporal (parcial, total ou ausência) e pela posição corporal adoptada (1). Este conjunto de características provoca diferenças substanciais na quantidade de massa muscular envolvida no exercício, no retorno venoso, na estimulação neural e no padrão de contracção muscular $(17,18)$. Na literatura internacional, têm sido vários os estudos realizados com o objectivo de diferenciar os ergómetros, a partir da análise de vários parâmetros fisiológicos em exercício realizado à mesma frequência cardíaca (FC). Contudo, estes estudos têm-se centrado apenas nas respostas agudas cardiovasculares ao exercício em steady-state realizado a 115bpm (5), a $65 \%$ da FC máxima real (22) e entre $60 \%$ e $70 \%$ da FC máxima teórica (19). Por outro lado, os protocolos utilizados pelos autores foram compostos por 15 minutos $(5,19)$ e por 60 minutos de exercício contínuo (22). Ou seja, não estão disponíveis estudos que caracterizem este tipo de respostas a percentagens mais elevadas de FC e, por outro lado, em vários patamares de exercício. Neste sentido, o objectivo do presente estudo foi o de conhecer as respostas cardiovasculares agudas a quatro modos de exercício (tapete, bicicleta, elíptica e remo), realizado em três patamares de exercício de forma incremental em intervalos compreendidos entre $60 \%$ e $85 \%$ da FC máxima teórica. Decorrente da popularidade da FC como indicador de controlo do esforço, este tipo de informação pode desempenhar um papel muito importante no processo de prescrição do exercício cardiovascular.

\section{MATERIAL E MÉTODOS}

\section{Amostra}

A amostra foi composta por 14 indivíduos voluntários do sexo feminino, fisicamente activos (alunos de uma Licenciatura em Educação Física e Desporto) e saudáveis. Nenhum era fumador e não se encontra- vam sob o efeito de qualquer medicamento. No Quadro 1 encontram-se as características da amostra em estudo.

\section{Procedimentos}

Antes de iniciarem o protocolo experimental, os sujeitos foram submetidos a uma sessão de esclarecimentos gerais, na qual assinaram um termo de consentimento para a participação neste estudo (de acordo com as normas do ACSM e a Declaração de Helsínquia de 1975), e a uma sessão prática de 40 minutos, para familiarização com quatro ergómetros (elíptica - Cyclone Cross-Trainner, tapete mecânico Proaction BH, remo - Concept II e bicicleta SensorMedics Medifit MFE 500U). O tempo da sessão prática foi subdividido em 10 minutos de exercício para cada ergómetro. Com o objectivo de diminuir a variação dos padrões técnicos do exercício em cada ergómetro, nas sessões de familiarização, os indivíduos foram instruídos a manter sempre o mesmo padrão (4). No tapete, com uma inclinação de $2 \%$ durante todo o protocolo experimental, foram instruídos para não se apoiarem (com o tronco para a frente) ou agarrarem a barra do ergómetro, mas apenas colocarem as mãos em cima do suporte, para facilitar o movimento $(4,27)$. Na elíptica, foram instruídos para levantar os calcanhares do apoio o menos possível (as características do ergómetro permitem localizar o esforço mais no músculo quadricípede ou nos MI no geral). Na bicicleta, foram instruídos para não ultrapassar as $60 \mathrm{rpm}$, uma vez que a cadência da pedalada pode influenciar o dispêndio energético (3) e a resposta cardio-vascular (9). Posteriormente, procedeu-se à realização de duas sessões de familiarização (durante a tarde) com o protocolo definido para este estudo (ver Quadro 2). 
Quadro 2. Protocolo experimental utilizado [tempos de repouso, de exercício e diferentes parâmetros avaliados].

\begin{tabular}{|c|c|c|c|c|c|c|c|c|}
\hline & Repouso & Intervalo & $1^{0}$ patamar & Intervalo & $2^{\circ}$ patamar & Intervalo & $3^{\circ}$ patamar & Final \\
\hline $\begin{array}{l}\text { Intensidade do } \\
\text { exercício [\% FCmáx.] }\end{array}$ & & & [60\%-65\%] & & [70\%-75\%] & & [80\%-85\%] & \\
\hline \multirow[t]{2}{*}{ Duração } & $5 \mathrm{~min}$ & $1 \mathrm{~min}$ & $5 \mathrm{~min}$ & $1 \mathrm{~min}$ & $5 \mathrm{~min}$ & $1 \mathrm{~min}$ & $5 \mathrm{~min}$ & $1 \mathrm{~min}$ \\
\hline & $\mathrm{VO}_{2}$ & TAS & $\mathrm{VO}_{2}$ & TAS & $\mathrm{VO}_{2}$ & TAS & $\mathrm{VO}_{2}$ & TAS \\
\hline Parâmetros & VE & TAD & VE & TAD & VE & TAD & VE & TAD \\
\hline Avaliados & QR & & $\mathrm{QR}$ & & QR & & $\mathrm{QR}$ & \\
\hline
\end{tabular}

Legenda: V02 $\rightarrow$ consumo de oxigénio; FCmáx. $\rightarrow$ frequência cardíaca máxima; TAS $\rightarrow$ tensão arterial sistólica; $T A D \rightarrow$ tensão arterial diastólica; $V \mathcal{E} \rightarrow$ ventilação; $Q R \rightarrow$ quociente respiratório.

Os indivíduos realizaram o mesmo protocolo para todos os ergómetros estudados. O protocolo experimental, para cada ergómetro utilizado, foi constituído por um período de repouso de cinco minutos (em posição erecta), imediatamente antes da realização de 3 patamares de exercício com intensidade crescente. Antes do início do protocolo (10 minutos) foi colocado, no sujeito a ser testado, um medidor de frequência cardíaca Polar (modelo Vantage NV), para que os intervalos de FC definidos fossem controlados. Este instrumento foi utilizado continuamente durante todo o protocolo experimental, onde se procedeu ao registo da FC de 5 em 5 segundos. Cada patamar teve a duração de 5 minutos e a intensidade do exercício (relativa à FC máxima teórica, calculada através da equação 220 -idade \pm 10 ) foi mantida entre $60 \%$ e $65 \%$ no primeiro patamar, $70 \%$ e $75 \%$ no segundo patamar e entre $80 \%$ e $85 \%$ no terceiro patamar. Entre cada patamar foi realizado um intervalo de inactividade de 1 minuto. No último minuto do período de repouso e no último minuto de cada um dos patamares, procedeu-se ao registo do consumo de oxigénio $\left(\mathrm{VO}_{2}\right)$ da ventilação pulmonar (VE) e do quociente respiratório $(\mathrm{QR})$. A tensão arterial sistólica (TAS) e a tensão arterial diastólica (TAD) foram medidas através de um esfigmomanómetro electrónico DINAMAP 8800 BP nos diferentes momentos do protocolo experimental apresentados no Quadro 2. Para a recolha de dados, os indivíduos da amostra acederam ao laboratório em dias diferentes (foram mantidas 48 horas de intervalo entre os momentos de recolha de dados), com o intuito de respeitar os tempos de recuperação para cada ergómetro. Todos os momentos da recolha de dados decorreram no período da tarde entre as 14:00 horas e as 17:30 horas (cada indivíduo foi testado sempre à mesma hora). Desta forma, cada indivíduo acedeu ao laboratório para a realização dos testes em 4 dias separados (um dia para cada ergómetro) e realizaram a totalidade dos testes no espaço de 12 dias. A sequência dos ergómetros para a realização do protocolo foi mantida para todos os indivíduos da amostra ( $1^{\circ}$ - elíptica, $2^{\circ}$ - tapete, $3^{\circ}$ - remo e $4^{\circ}$ - bicicleta). $\mathrm{O}$ $\mathrm{VO}_{2}$, a VE e o $\mathrm{QR}$, continuamente monitorizados através de um circuito aberto de espirometria (sistema SensorMedics 2900c), foram medidos de $20 \mathrm{em}$ 20 segundos e foi registada a média do último minuto do período de repouso e de cada patamar. A resistência dos ergómetros foi constantemente manipulada, de forma a cumprir com os intervalos de FC definidos. Estes procedimentos repetiram-se para todos os patamares subsequentes. A temperatura ambiente, a pressão atmosférica e o grau de humidade relativa foram idênticos em todos os momentos de recolha.

\section{Análise de dados}

Os resultados obtidos foram relativizados, para cada um dos ergómetros, aos valores de repouso (estabelecido em 100\%), no sentido de reduzir a variação intra e inter-individual. Posteriormente e a partir da variação destes valores, foram calculadas equações de regressão para cada variável em cada um dos ergómetros em estudo. Em cada patamar de exercício, as diferenças nos valores médios de cada variável dependente, em função do modo de exercício (ergómetro), foram testadas pelo recurso à análise de variância simples para medidas independentes (ANOVA). As comparações múltiplas foram realizadas pelo teste de Scheffé. O nível de significância foi mantido em $5 \%$. 


\section{RESULTADOS}

O Quadro 3 apresenta os valores absolutos das variáveis estudadas em cada momento do protocolo experimental e em cada ergómetro. Adicionalmente, apresentam-se os resultados do teste de diferenças de médias referentes aos valores relativizados ao de repouso e os resultados do correspondente teste de comparações múltiplas.

Quadro 3. Valores médios ( \pm desvio padrão) dos resultados absolutos e valores do teste de diferenças referente aos resultados relativizados ao valor de repouso.

\begin{tabular}{|c|c|c|c|c|c|c|c|}
\hline Variável & Patamar & Bicicleta & Elíptica & Remo & Tapete & $F_{(3,50)}$ & $P$ \\
\hline \multirow{4}{*}{$\begin{array}{l}\mathrm{VO}_{2} \text { relativo } \\
{[\mathrm{ml} / \mathrm{kg} / \mathrm{min}]}\end{array}$} & repouso & $5,77 \pm 1,1$ & $6,11 \pm 2,1$ & $5,39 \pm 2,1$ & $5,37 \pm 1,2$ & - & - \\
\hline & [60-65\%FCmáx.] & $18,75 \pm 4,0 \mathrm{e}$ & $16,39 \pm 5,7 \mathrm{btr}$ & $17,93 \pm 5,7 \mathrm{e}$ & $18,91 \pm 2,6 \mathrm{e}$ & 10,2 & $<0,001$ \\
\hline & [70-75\%FCmáx.] & $23,72 \pm 3,7$ e & $20,82 \pm 7,0 \mathrm{btr}$ & $22,04 \pm 7,1 \mathrm{e}$ & $23,90 \pm 3,0 \mathrm{e}$ & 10,3 & $<0,001$ \\
\hline & [80-85\%FCmáx.] & $29,20 \pm 3,5 \mathrm{e}$ & $25,24 \pm 9,0 \mathrm{btr}$ & $27,34 \pm 9,1 \mathrm{e}$ & $28,55 \pm 3,0 \mathrm{e}$ & 9,9 & $<0,001$ \\
\hline \multirow{4}{*}{$\begin{array}{l}\text { Ventilação } \\
\text { (I/min }]\end{array}$} & repouso & $11,98 \pm 2,0$ & $11,5 ? \pm 4,8$ & $11,30 \pm 3,8$ & $10,20 \pm 2,0$ & - & - \\
\hline & [60-65\%FCmáx.] & $30,23 \pm 7,1 \mathrm{r}$ & $27,85 \pm 6,0 \mathrm{tr}$ & $32,59 \pm 4,9 \mathrm{eb}$ & $28,33 \pm 5,4 \mathrm{e}$ & 9,5 & $<0,001$ \\
\hline & [70-75\%FCmáx.] & $38,85 \pm 7,8 \mathrm{r}$ & $35,78 \pm 7,7 \mathrm{tr}$ & $39,45 \pm 6,8 \mathrm{eb}$ & $36,15 \pm 5,3 e$ & 7,6 & $<0,001$ \\
\hline & [80-85\%FCmáx.] & $50,32 \pm 8,7$ & $45,54 \pm 11,3 r$ & $51,58 \pm 10,6$ e & $44,60 \pm 7,9$ & 6,7 & $<0,001$ \\
\hline \multirow{4}{*}{$\begin{array}{l}\text { Quociente } \\
\text { respiratório }\end{array}$} & repouso & $0,91 \pm 0,1$ & $0,77 \pm 0,2$ & $0,89 \pm 0,1$ & $0,87 \pm 0,1$ & - & - \\
\hline & [60-65\%FCmáx.] & $0,92 \pm 0,1$ et & $0,81 \pm 0,2 \mathrm{btr}$ & $0,90 \pm 0,1$ et & $0,86 \pm 0,1 \mathrm{ebr}$ & 547,8 & $<0,001$ \\
\hline & [70-75\%FCmáx.] & $0,96 \pm 0,1 \mathrm{et}$ & $0,83 \pm 0,3 \mathrm{btr}$ & $0,91 \pm 0,1 \mathrm{et}$ & $0,90 \pm 0,0 \mathrm{ebr}$ & 500,9 & $<0,001$ \\
\hline & [80-85\%FCmáx.] & $0,98 \pm 0,0$ et & $0,87 \pm 0,3 \mathrm{btr}$ & $0,95 \pm 0,1$ et & $0,93 \pm 0,0 \mathrm{ebr}$ & 430,3 & $<0,001$ \\
\hline \multirow{4}{*}{$\begin{array}{l}\text { Tensão arterial } \\
\text { sistólica } \\
{[\mathrm{mmHg}]}\end{array}$} & repouso & $\begin{array}{c}135,9 \pm 11, ? \\
\text { tre }\end{array}$ & $\begin{array}{c}146,2 \pm 13,8 \\
\text { rtb }\end{array}$ & $\begin{array}{c}140,8 \pm 14,1 \\
\text { tbe }\end{array}$ & $\begin{array}{c}134,4 \pm 16, ? \\
\text { ber }\end{array}$ & - & - \\
\hline & [60-65\%FCmáx.] & $\begin{array}{c}154,3 \pm 12,3 \\
\text { tre }\end{array}$ & $\begin{array}{c}145,6 \pm 12,6 \\
\mathrm{rtb}\end{array}$ & $\begin{array}{c}150,0 \pm 12,8 \\
\text { tbe }\end{array}$ & $150,0 \pm 9,5$ ber & 1738,7 & $<0,001$ \\
\hline & [70-75\%FCmáx.] & $\begin{array}{c}164,6 \pm 14,4 \\
\text { tre }\end{array}$ & $\begin{array}{c}158,9 \pm 12,4 \\
\text { rtb }\end{array}$ & $\begin{array}{c}154,9 \pm 10,0 \\
\text { tbe }\end{array}$ & $\begin{array}{c}160,5 \pm 11,4 \\
\text { ber }\end{array}$ & 1314,0 & $<0,001$ \\
\hline & [80-85\%FCmáx.] & $\begin{array}{c}165,9 \pm 16,2 \\
\text { tre }\end{array}$ & $\begin{array}{c}162,2 \pm 11,6 \\
\mathrm{rtb}\end{array}$ & $\begin{array}{c}161,4 \pm 12,7 \\
\text { tbe }\end{array}$ & $\begin{array}{c}162,2 \pm 18,3 \\
\text { ber }\end{array}$ & 845,7 & $<0,001$ \\
\hline \multirow{4}{*}{$\begin{array}{l}\text { Tensão arterial } \\
\text { diastólica } \\
(\mathrm{mmHg})\end{array}$} & repouso & $79,8 \pm 8,8$ & $82,3 \pm 8,3$ & $7 ?, 9 \pm 7,6$ & $75,4 \pm 13,3$ & - & - \\
\hline & [60-65\%FCmáx.] & $79,7 \pm 8,6$ tre & $74,6 \pm 12,1 \mathrm{rtb}$ & $79,5 \pm 8,9$ tbe & $69,9 \pm 10,7$ ber & 43216691,8 & $<0,001$ \\
\hline & [70-75\%FCmáx.] & $81,1 \pm 10,1$ tre & $85,2 \pm 5,8 \mathrm{rtb}$ & $81,2 \pm 8,0$ the & $72,9 \pm 11,9$ ber & $405>7350,3$ & $<0,001$ \\
\hline & [80-85\%FCmáx.] & $81,6 \pm 7,2$ tre & $82,8 \pm 7,5 \mathrm{rtb}$ & $79,9 \pm 7,6$ tbe & $71,8 \pm 11,4$ ber & 34813936,2 & $<0,001$ \\
\hline
\end{tabular}

Legenda: $b \rightarrow$ significativamente diferente da bicicleta; $e \rightarrow$ significativamente diferente da elíptica; $r \rightarrow$ significativamente diferente do remo; $t \rightarrow$ significativamente diferente do tapete.

Os valores do $\mathrm{VO}_{2}$ relativo foram mais elevados no tapete e mais baixos na elíptica. Foram identificadas diferenças estatisticamente significativas nos três patamares de exercício, entre todos os pares de ergómetros, com excepção da bicicleta vs. tapete, bicicle- ta vs. remo e tapete vs. remo. A variação dos valores do $\mathrm{VO}_{2}$ relativo ao peso corporal, nos três patamares de exercício e em cada um dos ergómetros estudados, está representada na Figura 1. 


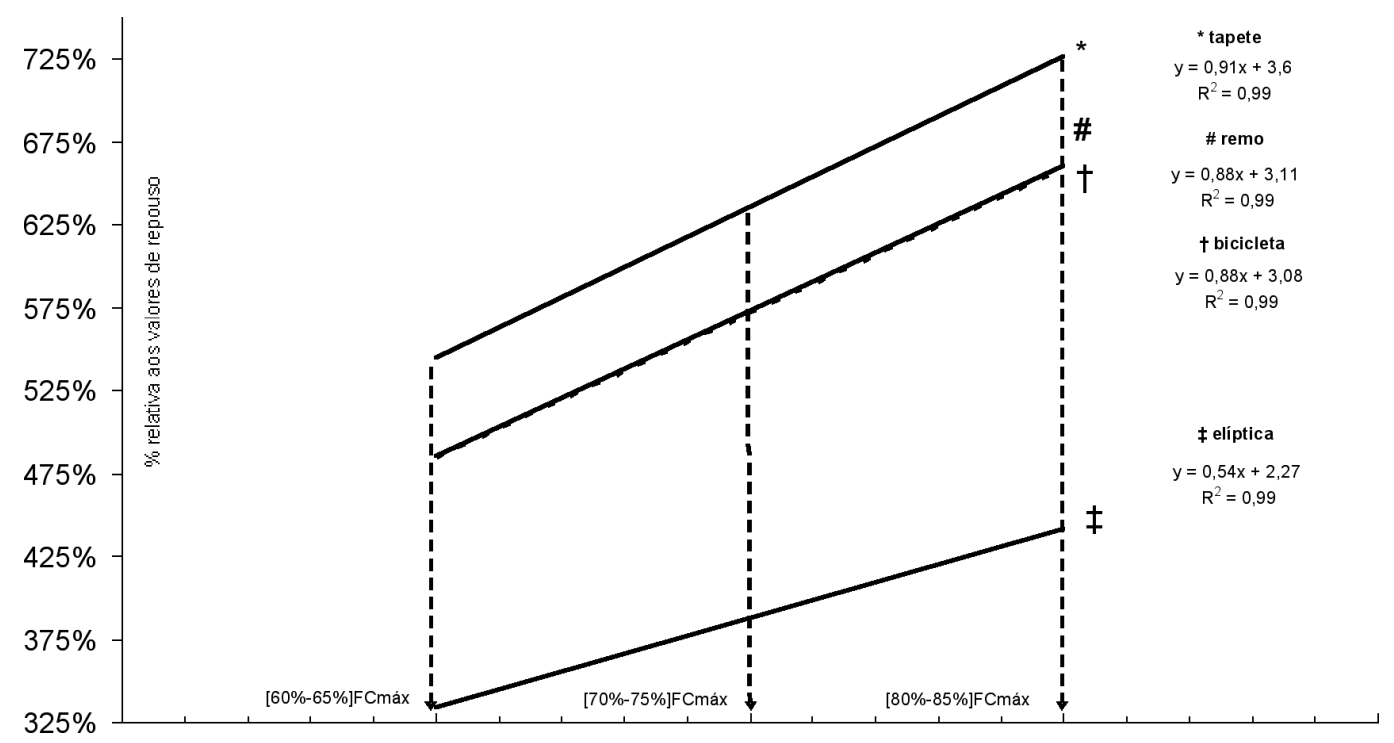

Figura 1. Variação dos valores médios do $\mathrm{VO}_{2}$ relativo, ao longo dos patamares, nos diferentes modos de exercício.

Os valores mais elevados do quociente respiratório foram obtidos na elíptica, enquanto que os valores mais baixos foram obtidos no tapete. Nos três patamares de exercício foram identificadas diferenças estatisticamente significativas entre todos os ergómetros, com excepção da bicicleta vs. remo. Na Figura 2 está representada a variação dos valores do quociente respiratório em cada um dos ergómetros estudados, nos três patamares de exercício.

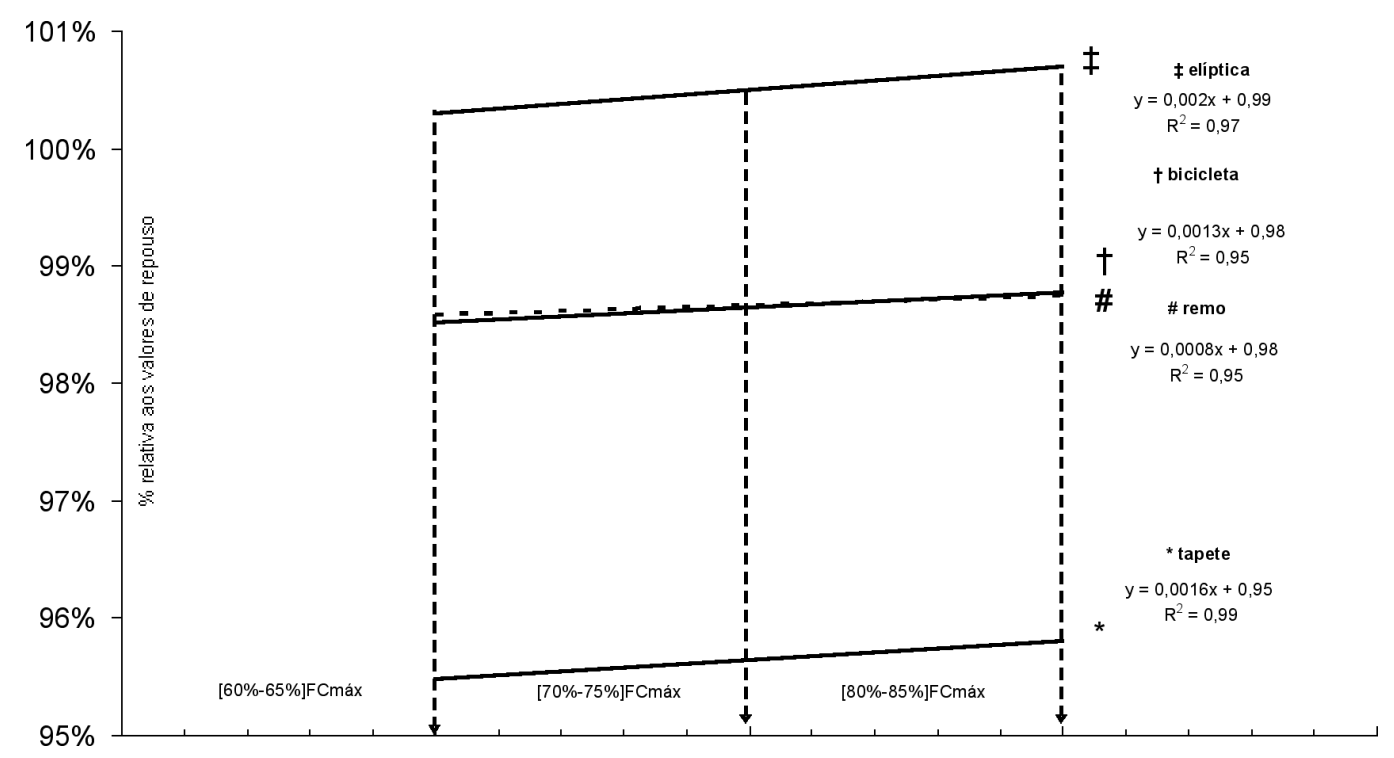

Figura 2. Variação dos valores médios do quociente respiratório, ao longo dos patamares, nos diferentes modos de exercício. 
Os valores da ventilação foram mais elevados no remo e mais baixos na elíptica. Nos três patamares de exercício foram identificadas diferenças estatisticamente significativas entre a elíptica vs. remo. As diferenças entre a elíptica vs. tapete e bicicleta vs. remo só tiveram significado estatístico no $1^{\circ}$ e $2^{\circ}$ patamares. O comportamento dos valores da ventilação em cada um dos ergómetros e patamares de exercício estudados está representado na Figura 3.

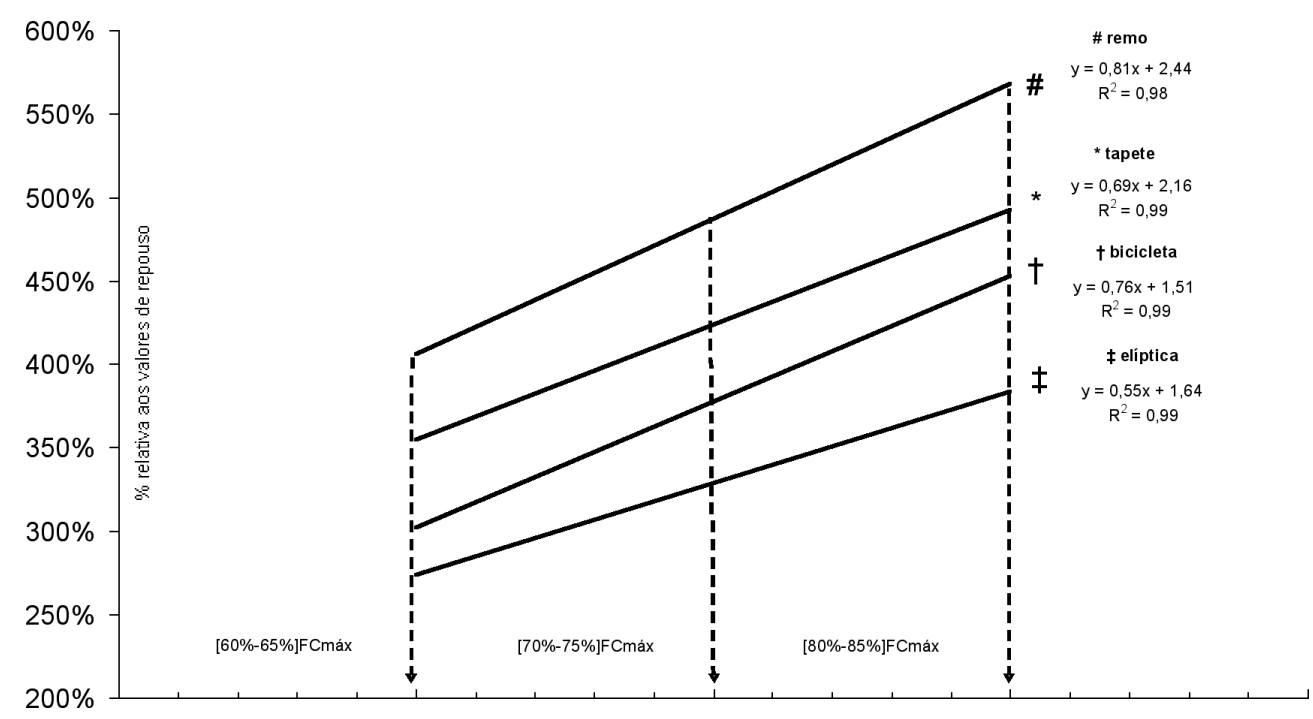

Figura 3. Variação dos valores médios da ventilação pulmonar, ao longo dos patamares, nos diferentes modos de exercício.

Os valores de tensão arterial sistólica foram mais elevados na bicicleta e no tapete e mais baixos na elíptica e no remo. Foram identificadas diferenças estatisticamente significativas nos três patamares de exercício entre todos os pares de ergómetros. O comportamento dos valores da tensão arterial sistólica em cada um dos ergómetros e patamares de exercício estudados está representado na Figura 4.

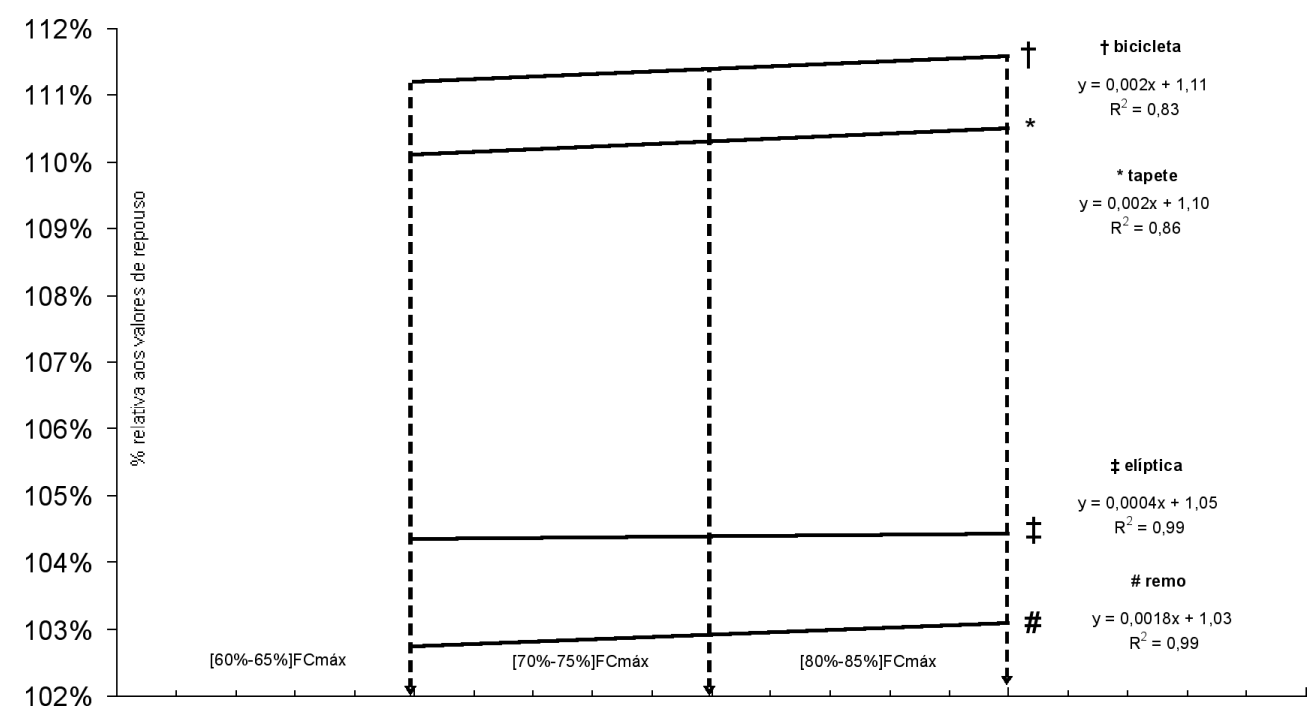

Figura 4. Variação dos valores médios da tensão arterial sistólica, ao longo dos patamares, nos diferentes modos de exercício. 
Os valores de tensão arterial diastólica foram mais elevados no tapete e mais baixos na bicicleta. Foram identificadas diferenças estatisticamente significativas nos três patamares de exercício entre todos os pares de ergómetros. O comportamento dos valores da tensão arterial diastólica em cada um dos ergómetros e patamares de exercício estudados está representado na Figura 5.

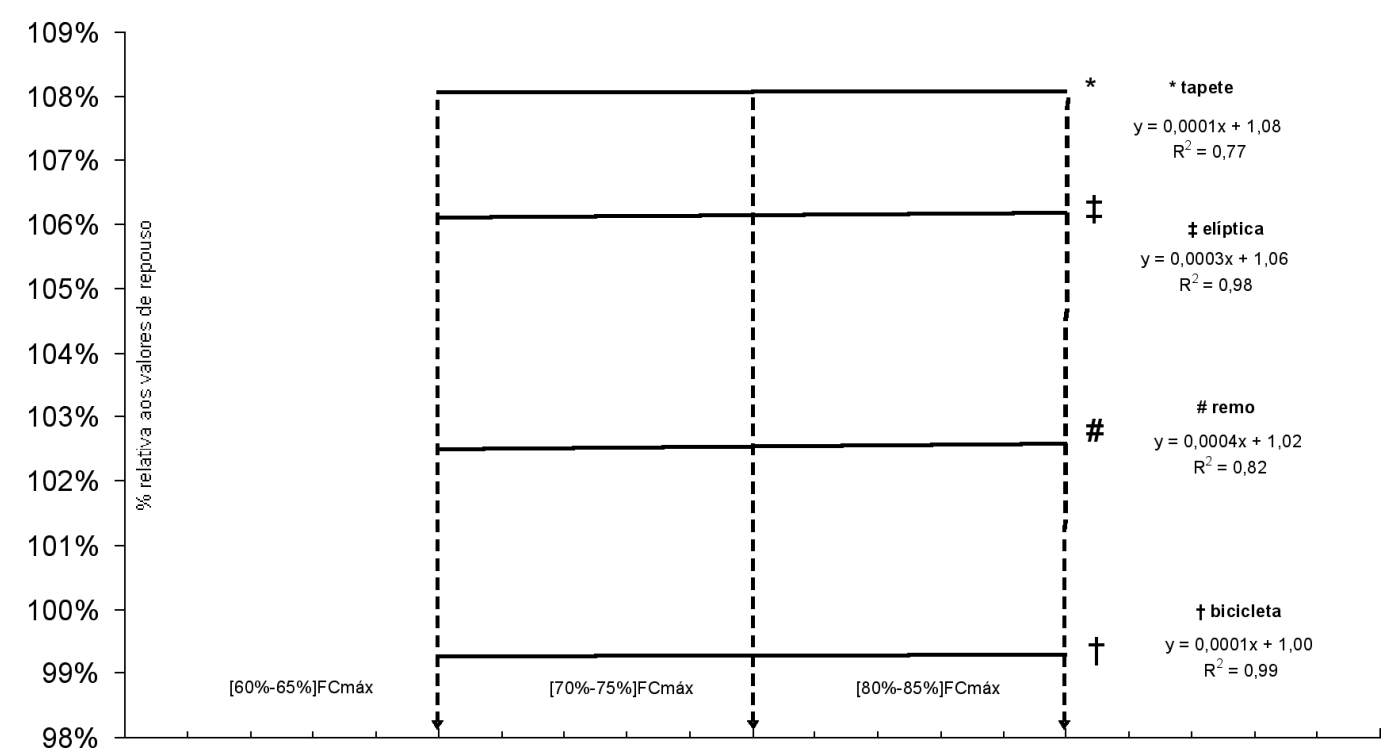

Figura 5. Variação dos valores médios da tensão arterial diastólica, ao longo dos patamares, nos diferentes modos de exercício.

\section{DISCUSSÃO}

Ao longo dos patamares de esforço e para todos os modos de exercício, os valores do $\mathrm{VO}_{2}$ aumentaram linearmente com o aumento da FC. Apesar de ser um comportamento esperado, devido à forte relação existente entre a $\mathrm{FC}$ e o $\mathrm{VO}_{2}$ neste tipo de esforço $(4,20,26)$, a magnitude deste aumento variou em função do modo de exercício. Neste sentido, parece claro que as características dos diferentes ergómetros influenciam a relação $\mathrm{VO}_{2} / \mathrm{FC}$. O pulso de oxigénio expressa a eficiência do sistema de transporte de oxigénio e indica-nos a quantidade de $\mathrm{O}_{2}$ consumida em cada batimento. Ou seja, para o mesmo esforço do miocárdio registaram-se consumos de oxigénio diferentes em função do modo de exercício. $\mathrm{O}$ tapete foi o ergómetro onde se registaram valores mais elevados de $\mathrm{VO}_{2}$ para a mesma FC. Do ponto de vista fisiológico, estes resultados têm sido explicados pela elevada quantidade de massa muscular solicitada durante o exercício de MI, pelo facto do aumento na solicitação de massa muscular implicar maior exigência de oxigénio $(4,6,15,23,27)$. A esta razão acresce-se o facto do indivíduo suportar o seu peso corporal, situação que vai implicar um padrão de contracção muscular distinto (carga mais elevada) e a manutenção do centro de gravidade estável (maior solicitação dos músculos de suporte). No presente estudo, apesar do protocolo ter sido realizado com apoio nas barras do tapete, o que parece implicar valores inferiores de aproximadamente $0,5 \mathrm{l} / \mathrm{min}$ no $\mathrm{VO}_{2}$ e $10 \mathrm{bpm}$ na FC (4), foi utilizado um tapete mecânico. Esta característica, motivando um padrão de contracção muscular mais exigente para mover a passadeira, tende a exagerar os valores do $\mathrm{VO}_{2}(8,10)$. Por outro lado, apesar dos elementos da amostra não apresentarem especializações nos modos de exercício realizados, parece inequívoco que o exercício realizado no tapete é o mais 
familiar e, desta forma, o que poderá induzir melhores prestações.

Os valores do $\mathrm{VO}_{2}$ registados no remo foram muito idênticos aos registados na bicicleta. Entre estes dois ergómetros não existem diferenças no suporte do peso corporal; no entanto, a solicitação de massa muscular no remo é mais elevada (porque solicita MS e MI). Desta forma, seria de esperar que os valores do $\mathrm{VO}_{2}$ no remo se apresentassem mais elevados (14). Todavia, é consensual que a resposta cardio-respiratória ao exercício que inclua MS é mais acentuada relativamente ao exercício de MI, devido ao aumento da resposta do sistema nervoso simpático, com alteração da distribuição do fluxo sanguíneo $(12,13,15)$. Ou seja, para o mesmo valor de $\mathrm{VO}_{2}$, a FC é mais elevada no modo que solicita MS. O inverso também pode ser colocado. Para a mesma $\mathrm{FC}, \mathrm{o} \mathrm{VO}_{2}$ é inferior para o modo que solicita MS. Neste sentido, quando a intensidade do exercício é controlada pela FC (como no presente estudo) parece possível que os valores do $\mathrm{VO}_{2}$ obtidos no remo e na bicicleta sejam muito idênticos.

A elíptica foi o ergómetro onde se registaram valores significativamente mais baixos no $\mathrm{VO}_{2}$. De forma idêntica ao remo, a elíptica solicita MI e MS; no entanto, neste ergómetro os indivíduos suportam o seu peso corporal. Contudo, o envolvimento dos MS nos gestos técnicos destes dois ergómetros, determinado pelas características mecânicas dos engenhos, apresenta diferenças substanciais. Enquanto que no remo o envolvimento dos MS é particularmente activo (pela "puxada"), na elíptica, como as barras de suporte dos MS estão interligadas com os pedais dos MI (cujo movimento determina o movimento das barras de suporte dos MS), o trabalho realizado pelos MS é determinado em função do trabalho realizado pelos MI. Desta forma, os MS parecem não contribuir com grande relevância para o trabalho total realizado, facto que pode explicar os valores de $\mathrm{VO}_{2}$ mais baixos (17). Por outro lado, os valores do remo sugerem que a intervenção dos MS foi superior, contribuindo em maior magnitude para o trabalho total realizado.

Devido às suas características, nestes dois ergómetros (remo e elíptica), não foi possível medir a percentagem de trabalho realizado com os MS e MI, facto que dificulta a análise dos resultados.
A partir destes resultados e da literatura disponível, é igualmente possível verificar que os modos de exercício com envolvimento dos MS e MI não parecem muito associados a melhores pulsos de $\mathrm{O}_{2}$. Ou seja, para FC idênticas, nestes modos de exercício o $\mathrm{VO}_{2}$ é inferior (22).

Para todos os modos de exercício foi identificado um aumento da VE em função do aumento da FC (i.e., do aumento da intensidade do esforço). Para qualquer tipo de exercício, com o aumento da intensidade, as concentrações de $\mathrm{CO}_{2}$ e de iões de hidrogénio no sangue venoso aumentam também, estimulando os quimiorreceptores e promovendo um aumento ventilatório $(4,20)$. Para além destes, os factores neuronais aferentes ao centro respiratório também deverão ter contribuído para este aumento da ventilação. A partir dos resultados obtidos, foi possível identificar valores da VE significativamente mais elevados no remo. Os valores da VE deveriam apresentar tendências semelhantes às identificadas para os valores do $\mathrm{VO}_{2}$. Ou seja, os ergómetros com valores de $\mathrm{VO}_{2}$ mais elevados deveriam apresentar VE mais elevadas. De facto, no exercício em steady-state a VE aumenta de forma linear com o $\mathrm{VO}_{2}$, de forma a manter os níveis alveolares da pressão de $\mathrm{O}_{2}$ e de $\mathrm{CO}_{2}$ próximos dos valores de repouso $(7,21,26)$. Este facto, só não se verificou no remo, onde a VE foi a mais elevada; contudo, foi bem patente nos restantes ergómetros. Buckley, Davies e Simpson (6) verificaram que os valores do limiar ventilatório e do limiar láctico foram atingidos a intensidades inferiores no remo, comparativamente com o tapete. Esta constatação pode ser justificada pela posição corporal que os indivíduos têm que adoptar no remo. Ou seja, o "movimento normal" dos músculos ventilatórios é condicionado e limita a maximização da ventilação $(16,25)$. Neste sentido, para que se possa manter a intensidade do exercício, torna-se necessário aumentar a VE. Este quadro de argumentos parece explicar os elevados valores de VE e a elevada importância do treino dos músculos respiratórios nos remadores de elite (25). Os valores mais elevados da VE no remo, podem igualmente ser justificados pelo recrutamento de fibras musculares tipo II. A solicitação deste tipo de fibras promove o aumento das concentrações de lactato sanguíneo o qual, quando não é eliminado, proporciona uma acumula- 
ção de iões de hidrogénio com aumento da VE. Por outro lado, a partir da literatura disponível é possível perceber que, para o mesmo $\mathrm{VO}_{2}$ e carga externa, os valores da VE no exercício de MS são superiores aos identificados no exercício de MI $(4,26)$ e os valores obtidos em exercício combinado (MS e MI) são ainda mais elevados $(17,18)$. Este facto está também patente nos resultados do presente estudo. Apesar dos valores do $\mathrm{VO}_{2}$ na bicicleta e no remo terem sido muito idênticos nos três patamares de esforço, o exercício realizado no remo (solicitação de MS e MI) induziu valores de VE significativamente superiores aos obtidos na bicicleta.

Nos valores do QR foram identificadas diferenças estatisticamente significativas entre todos os pares de ergómetros que foram comparados $(\mathrm{p}<0,05)$, com excepção dos valores registados na bicicleta vs. remo. Os valores do $Q R$ foram superiores na elíptica e inferiores no tapete.

$\mathrm{O} Q R$ é um indicador que expressa indirectamente a comparticipação dos substratos energéticos durante o exercício $(21,26)$. Os valores obtidos na elíptica (mais elevados) sugerem a utilização de maior percentagem de hidratos de carbono, facto directamente associado a uma maior solicitação de fibras tipo II. Por outro lado, os valores obtidos no tapete parecem sugerir o inverso, ou seja, a utilização de maior percentagem de gorduras (maior solicitação de fibras tipo I).

Os estudos disponíveis neste domínio parecem consensuais. Thomas, Feiock e Araujo (22) identificaram valores de QR ligeiramente superiores (sem diferenças estatisticamente significativas) nos ergómetros onde os indivíduos não suportavam o seu peso corporal (remo e bicicleta) comparativamente com o tapete e o simulador de ski. Buckley, Davies e Simpson (6) registaram diferenças estatisticamente significativas nos valores do $\mathrm{QR}$ entre o remo e o tapete $(0,99 \pm 0,07$ e $0,94 \pm 0,07$, respectivamente). No remo, o limiar ventilatório e o limiar láctico foram atingidos a intensidades inferiores comparativamente com o tapete. Os autores justificam estes resultados pela diferente constituição das fibras musculares dos MI e MS e pelas diferenças no seu recrutamento em cada modo de exercício. No entanto, a especificidade da amostra na realização do exercício desempenha um papel igualmente importante. Zeni, Hoffman e Clifford (27) e Buckley, Davies e
Simpson (6) referem que indivíduos com pouca experiência no remo, concentram mais acção (força) nos braços, comparativamente com remadores experientes. Acrescentam que, mesmo quando a percentagem de $\mathrm{VO}_{2}$ máximo é inferior no remo, as concentrações de lactato são superiores relativamente ao tapete. Ou seja, é provável que se obtenham melhores prestações (e mais económicas do ponto de vista glicolítico) em movimentos mais familiares, facto que pode explicar os valores de lactato sanguíneo mais baixos no tapete e mais elevados na elíptica. Nos valores da TAS, a transição do patamar de repouso para o primeiro patamar de esforço foi caracterizada por um aumento acentuado. Nos patamares subsequentes os valores aumentaram muito ligeiramente (ver Figura 4). Estes resultados permitiram identificar diferenças estatisticamente significativas entre os valores de todos os modos de exercício (ver Quadro 3). Os valores de TAS foram mais elevados nos ergómetros de MI (bicicleta e tapete), comparativamente aos que solicitam MS e MI (elíptica e remo).

Enquanto que os valores da tensão arterial sistólica são influenciados fundamentalmente pela intensidade do exercício, os valores da tensão arterial diastólica são mais influenciados pela quantidade de massa muscular solicitada (26). Desta forma, o efeito da massa muscular solicitada na TAS decorre fundamentalmente da associação entre a TAS e a TAD. A maiores intensidades do exercício correspondem valores de tensão arterial sistólica mais elevados, facto decorrente do aumento do débito cardíaco e das alterações na actividade do sistema nervoso simpático $(4,7)$. De facto, para a mesma FC, no tapete e na bicicleta (modos de exercício com valores de $\mathrm{VO}_{2}$ mais elevados e com menor solicitação de massa muscular) registaram-se valores de TAS mais elevados. Por outro lado, a maiores quantidades de massa muscular solicitada correspondem TAS mais baixas. Por exemplo, o exercício realizado no remo induziu, nos três patamares de esforço, valores de $\mathrm{VO}_{2}$ similares aos registados na bicicleta $(\mathrm{p}=$ n.s.). Contudo, a solicitação acrescida de massa muscular no remo (MI e MS) induziu valores de TAS mais baixos. $\mathrm{Na}$ literatura, os estudos centrados na resposta aguda deste parâmetro aos diferentes modos de exercício (em populações normotensas) são quase omissos. 
Aminoff, Smolander, Korhonen e Louhevaara (2) compararam os resultados entre exercício realizado a $50 \%$ e a $75 \%$ do $\mathrm{VO}_{2}$ num ergómetro de braços e numa bicicleta; no entanto, apenas mediram a TAS na bicicleta.

Os valores da TAD aumentaram do patamar de repouso para o primeiro patamar de esforço em todos os ergómetros, com excepção da bicicleta (ver Figura 5). No entanto, a magnitude deste aumento variou significativamente em função do modo de exercício. Após o primeiro patamar de esforço, os valores da TAD, atingidos em cada modo de exercício, mantiveram-se estáveis até ao último patamar de esforço. Como na base do controlo da tensão arterial estão reflexos neurais que influenciam directamente a actividade do centro vasomotor (11), a elevação tensional promove a distensão e excitação dos receptores neurais (barorreceptores), com inibição da actividade do centro vasomotor. Desta forma, diminuem a FC e o volume sistólico, no sentido de diminuir a TA (11). Quando os valores da tensão arterial estão demasiado baixos, os barorreceptores deixam de ser estimulados, excitando o centro vasomotor que leva os valores da tensão arterial ao normal (11). A maiores quantidades de massa muscular solicitada estão associados valores de TAD mais baixos. Esta variação ocorre porque associado ao exercício com grande quantidade de massa muscular activa está uma elevada dilatação dos vasos sanguíneos e uma diminuição das resistências vasculares periféricas, devida ao controlo do diâmetro de abertura capilar pela acção dos esfíncteres pré-capilares (7). No entanto, a quantidade de massa muscular solicitada não é o único factor determinante destas diferenças. Caso contrário, os valores de TAD seriam mais elevados na bicicleta e no tapete (porque solicitam menos massa muscular). De facto, os valores (de TAD) obtidos no presente estudo foram mais elevados nos modos de exercício onde o indivíduo suporta o seu peso corporal e está numa posição vertical (tapete e elíptica) e mais baixos nos modos onde o ergómetro suporta o peso dos indivíduos (remo e bicicleta).

Esta tendência nos valores do tapete pode ser justificada, do ponto de vista metodológico, porque no presente estudo foi utilizado um tapete mecânico (Proaction BH). De facto, esta característica particular parece influenciar, de forma determinante, a resposta aguda da TAD. A maior resistência a vencer para mover a passadeira do tapete mecânico exige mais força. Este facto parece promover um ciclo de marcha envolvendo maiores pressões intersticiais nos músculos solicitados, o que pode levar ao aumento das resistências vasculares periféricas e, consequentemente, aumentar os valores da TAD.

Os resultados do presente estudo sugerem que os ergómetros que solicitam MS e MI (para a mesma FC) induzem TAS mais baixas ao longo dos patamares de esforço. Elevadas tensões arteriais sistólicas estão associadas a maiores índices de stress do sistema cárdio-vascular e a maiores sobrecargas do miocárdio $(7,21,26)$. Por outro lado, os valores mais baixos ao longo dos intervalos de esforço na TAD ocorreram nos ergómetros em que os indivíduos estão sentados. Turley e Wilmore (24) confirmam esta tendência nos resultados e justificam-nos pelos menores débitos cardíacos e menores resistências vasculares periféricas.

\section{CONCLUSÕES}

Da análise realizada aos resultados obtidos destacam-se as seguintes conclusões:

- Os valores do $\mathrm{VO}_{2}$ aumentaram linearmente ao longo dos patamares de esforço, em todos os modos de exercício. A magnitude deste aumento variou em função das diferenças na quantidade de massa muscular envolvida no exercício e no tipo de suporte do peso corporal. Os valores mais elevados foram registados no tapete, enquanto que os valores mais baixos foram registados na elíptica.

- Para todos os modos de exercício foi identificado um aumento da VE ao longo dos patamares. O remo foi o ergómetro onde se registaram valores mais elevados, facto que pode decorrer da posição corporal que os indivíduos têm que adoptar, do recrutamento de fibras musculares tipo II e da combinação de exercício de MS e MI.

- A elíptica foi o ergómetro com valores de QR mais elevados, o que sugeriu a utilização de maior percentagem de hidratos de carbono, provavelmente explicado pela maior solicitação de fibras tipo II. Por outro lado, os valores obtidos no tapete sugeriram a utilização de maior percen- 
tagem de gorduras, passível de ser explicado pela maior solicitação de fibras tipo I.

- Foi possível identificar um aumento na TAS ao longo dos patamares de exercício. A tendência dos resultados sugere valores mais elevados nos ergómetros que solicitam menor quantidade de massa muscular (apenas MI - bicicleta e tapete).

- Os valores da TAD foram mais elevados nos modos de exercício onde o indivíduo suporta o seu peso corporal e está numa posição vertical (tapete e elíptica) e mais baixos nos modos onde o ergómetro suporta o peso dos indivíduos (remo e bicicleta).

Para a mesma percentagem de frequência cardíaca máxima, as repercussões cardio-respiratórias aos diferentes modos de exercício realizados apresentaram diferentes perfis de resposta aguda. Este tipo de resposta parece depender da quantidade de massa muscular envolvida no exercício, do suporte do peso corporal e do tipo de unidades motoras solicitadas. Apesar dos diversos ergómetros disponíveis permitirem atender ao gosto individual (e promoverem a adesão ao exercício), os cuidados no ajuste da prescrição do exercício aos objectivos e características do indivíduo são imperativos.

\section{CORRESPONDÊNCIA}

\section{Catarina Isabel N.G. Abrantes}

Departamento de Desporto

Universidade de Trás-os-Montes e Alto Douro

Rua Dr. Manuel Cardona

5000 Vila Real

PORTUGAL

abrantes@utad.pt 


\section{BIBLIOGRAFIA}

1 ACSM - American College of Sports Medicine (2000). Manual de Consulta para el Control y la Prescripción de Exercicio. Barcelona: Editorial Paidotribo

2 Aminoff T, Smolander J, Korhonen O, Louhevaara V (1997). Cardiorespiratory and subjective responses to prolonged arm and leg exercise in healthy young and older men. Eur J Appl Physiol 75: 363-368

3 Brisswalter J, Hausswirth C, Smith D, Vercruyssen F, Vallier J (1999). Energetically optimal cadence vs. freelychosen cadence during cycling: effect of exercise duration. Int J Sports Med 20: 60-64

4 Brooks G, Fahey T, White T (1996). Exercise Physiology Human Bioenergetics and its Applications. Mountain View: Mayfield Publishing Company

5 Brown S, Wu Q, Li H, Mao X (1994). Cardiorespiratory responses to low-intensity heart rate-controlled exercise in female subjects. J Sports Med Physical Fitness 34: 279-283

6 Buckley J, Davis J, Simpson T (1999). Cardiorespiratory responses to rowing ergometry and treadmill exercise soon after myocardial infarction. Med Sci Sports Exerc 31: 1721

7 Chicharro J, Vaquero A (1995). Fisiología del Ejercicio. Madrid: Editorial Médica Panamericana

8 Davies B, Daggett A, Jakeman P, Mulhall J (1984). Maximum oxygen uptake using different treadmill protocols. Br J Sports Med 18: 74-79

9 Deschenes M, Kraemer W, McCoy R, Volek J, Turner B, Weinlein J (2000). Muscle recruitment patterns regulate physiological responses during exercise of the same intensity. Am J Physiol 279: R2229-R2236

10 Gamble D, Jakeman P, Bartlett R (1987). A comparison of non-motorised treadmill, motorised treadmill and overground running patterns. In: Biomechanics in Sport. London: Institution of Mechanical Engineers, 25-32

11 Guyton A (1988). Fisiologia Humana. Rio de Janeiro: Guanabara Koogan

12 Harms C (2000). Effect of skeletal muscle demand on cardiovascular function. Med Sci Sports Exerc 32: 94-99

13 Hoffman M, Kassay K, Zeni A (1996). Does the amount of exercising muscle alter the aerobic demand of dynamic exercise? Eur J Appl Physiol 74: 541-547

14 Katsanos C, Cheuvront S, Haymes E (2001). Energy expenditure relative to perceived exertion: stationary cycling versus treadmill walking. Res Q Exerc Sport 72: 176-181

15 Kravitz L, Robergs R, Heyward V, Wagner D, Powers K (1997). Exercise mode and gender comparisons of energy expenditure at self-selected intensities. Med Sci Sports Exerc 29: 1028-1035

16 Mahler D, Andrea B, Ward J (1987). Comparison of exercise performance on rowing and cycle ergometers. Res $Q$ Exerc Sport 58: 41-46

17 Mayo J (1998). The Physiological Effects of Varied Arm and Leg Work During Total Body Exercise. Doctoral Dissertation, University of Mississipi

18 Mayo J, Kravitz L, Chitwood L, Kinzey S, Waters W, Wongsathikun J (1999). Cardiovascular responses to combine arm and leg exercise. Med Sci Sports Exerc 31: S421

19 Plunk J, Stephen F, Cantu R, Sherman N (2000). Caloric expenditure differences between individuals exercising on a motor driven treadmill versus stairclimber. Res $Q$ Exerc Sport 71: A33
20 Powers S, Howley E (2000). Fisiologia do Exercício: Teoria e Aplicação ao Condicionamento e ao Desempenho. São Paulo: Editora Manole

21 Robergs R, Roberts S (1997). Exercise Physiology: Exercise, Performance, and Clinical Applications. New York: Times Mirror-Mosby College Publishing

22 Thomas T, Feiock C, Araujo J (1989). Metabolic responses associated with four modes of prolonged exercise. J Sports Med Physical Fitness 29: 77-82

23 Thomas T, Ziogas G, Smith T, Zhang Q, Londeree B (1995). Physiological and perceived exertion responses to six modes of submaximal exercise. Res Q Exerc Sport 66: 239-246

24 Turley K, Wilmore J (1997). Cardiovascular responses to treadmill and cycle ergometer exercise in children and adults. J Appl Physiol 83: 948-957

25 Volianitis S, McConnell A, Koutedakis Y (2001). Inspiratory muscle training improves rowing performance. Med Sci Sports Exerc 33: 803-809

26 Wilmore J, Costill D (2001). Fisiologia do Esporte e do Exercício. São Paulo: Editora Manole

27 Zeni A, Hoffman M, Clifford P (1996). Energy expenditure with indoor exercise machines. JAMA 275: 1424-1427. 